\title{
Quantitative Elemental Mapping with Electron Microprobe and Automated Data Analysis
}

\author{
Julie Chouinard $^{1}$ and John Donovan ${ }^{1}$ \\ 1. University of Oregon, CAMCOR, Eugene, OR, USA
}

While the utility of quantitative chemical mapping with electron microprobe (EPMA) can be overshadowed by the instrument time required to obtain such data, advancements in data acquisition and background corrections have made it a more practicable technique. The use of mean atomic number (MAN) background corrections can drastically shorten total run time by allowing the user to omit offpeak elemental maps by modeling the background for those elements instead [1]. Typically MAN corrections are limited to major elements, however, for simple matrices, a blank correction can be used in combination with MAN background to map trace elements, attaining similar accuracy to off-peak measurements while improving precision [2]. The advantages of mapping samples over doing single spot analyses are obvious: small scale chemical variations can be elucidated and differentiated from large scale features, for instance those seen in mineral grains with complex growth patterns; chemical information for phases proximate to those of interest can be later employed for determining the paragenesis of a rock sample or explaining away an apparent diffusion curve really attributable to secondary fluorescence; average compositions can be ascertained for materials that are heterogeneous at the micro- to millimeter scale; and countless other ways.

The use of customizable and automated scripts can greatly aid in quickly extracting useful and often supplemental information from of these quantitative maps; these can be produced with programming languages and software packages such as Golden Software's Surfer ${ }^{\circledR}$ (modified Visual Basic), R, and MathWorks' MATLAB ${ }^{\circledR}$. Several scripts have been made using the Scriptor application in Surfer ${ }^{\circledR}$ to automate and increase the speed of the analysis of mapped chemical data. To determine the composition over a diffusion boundary with a heterogeneous matrix, a script was crafted to average horizontal or vertical strips across a map [3]. Another was created to calculate the average composition of any delineated area, which can be used for defining the chemistry of a particular phase or reaction rim (Fig. 1). Scripts have also been generated to plot out the chemical changes along any traverse chosen on the map, such as [4]. Beyond chemical maps, programmable scripts have been developed to more efficiently process and examine spot analyses as well and several of these will be presented as well.

The addition of acquisition and analysis features already available for single spot analyses will further improve EPMA mapping capabilities, decrease run time, and ultimately increase its usage as it becomes more cost effective. The ability to acquire quantitative, background and matrix corrected, energy dispersive $\mathrm{x}$-day (EDX) maps for major elements simultaneous with wavelength dispersive $\mathrm{x}$-ray (WDX) maps for trace elements is most notably absent. While interferences are still an issue for some EDX analyses, for many major elements it can provide reliable data with satisfactory precision and accuracy; combining EDX and WDX is already relatively commonplace for single point analyses as it often does not add any time to the run because the data can be compiled concurrently. Another acquisition option that would enhance mapping capabilities is the introduction of the time dependent intensity element corrections, without it elements like sodium $(\mathrm{Na})$ that volatize and migrate in some matrices cannot be reliably mapped (Fig. 2). 


\section{References:}

[1] J Donovan and T Tingle, Journal of Microscopy and Microanalysis 2 (1996) p. 1.

[2] J Donovan and J Armstrong, Microscopy and Microanalysis 20 S3 (2014) p. 724.

[3] J Barkman et al, Microscopy and Microanalysis 19 S2 (2013) p. 848.

[4] J Chouinard et al, Microscopy and Microanalysis 20 S3 (2014) p. 750.

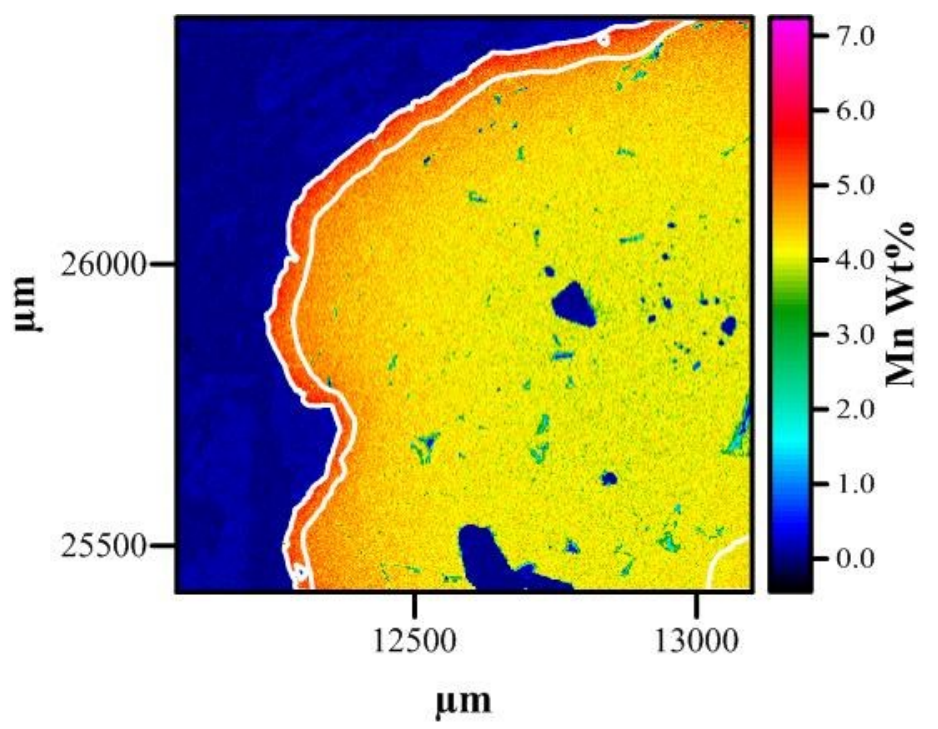

Figure 1. Quantitative map of manganese (Mn) in a garnet; the average weight percent of $\mathrm{Mn}$ in rim of garnet (outer edge outlined in white) is $5.19 \%$, compared to $4.22 \%$ in the core (bottom right corner outlined in white).

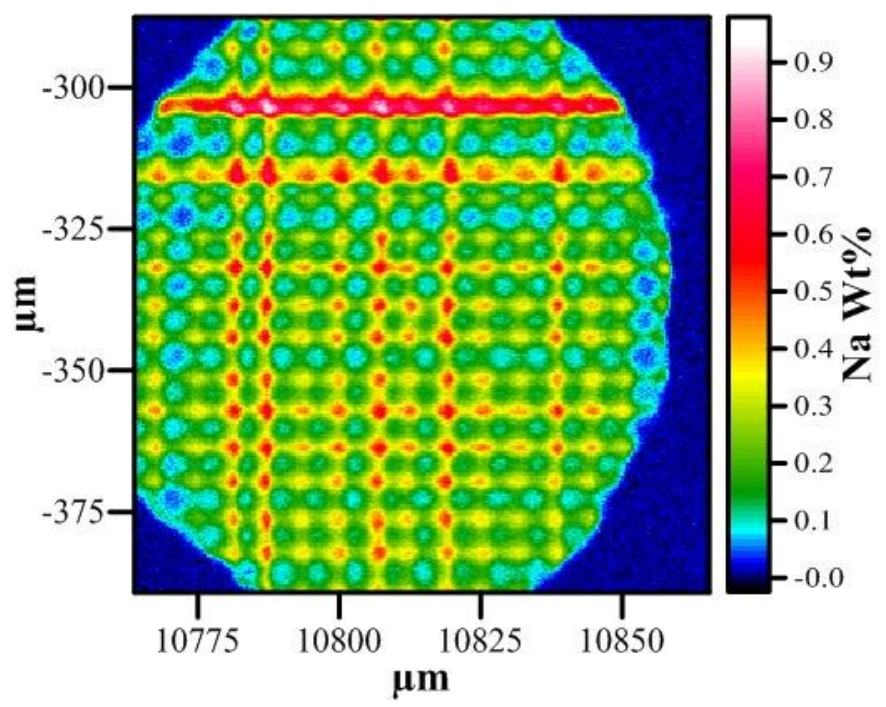

Figure 2. Map of the Na concentration in a melt inclusion hosted by olivine [4]. The pattern is believed to be the result of $\mathrm{Na}$ migration in the glass and not a real chemical gradient (it was not seen in any other element analyzed); the integration of TDI into mapping may help resolve this issue. 